Research Article

\title{
Flocculation Performance and Kinetics of Magnetic Polyacrylamide Microsphere under Different Magnetic Field Strengths
}

\author{
Wentian Yu, ${ }^{1}$ Chen Wang $\mathbb{D}^{1}{ }^{1}$ Gengliang Wang $\mathbb{D}^{2}{ }^{2}$ and Qing Feng ${ }^{1}$ \\ ${ }^{1}$ School of Environmental Science and Engineering, Qilu University of Technology (Shandong Academy of Sciences), \\ Jinan 250353, China \\ ${ }^{2}$ School of Mathematics and Statistics, Qilu University of Technology (Shandong Academy of Sciences), Jinan 250353, China \\ Correspondence should be addressed to Chen Wang; shanqing123@126.com
}

Received 22 May 2020; Revised 3 August 2020; Accepted 5 August 2020; Published 24 August 2020

Academic Editor: Pedro M. Mancini

Copyright (C) 2020 Wentian Yu et al. This is an open access article distributed under the Creative Commons Attribution License, which permits unrestricted use, distribution, and reproduction in any medium, provided the original work is properly cited.

In this study, the flocculation performance and kinetics of magnetic cationic polyacrylamide (MCPAM) microspheres, compared with cationic polyacrylamide (CPAM), were systematically investigated under different magnetic field strengths. Flocculation performance was observed by jar test experiment. The density of flocs was estimated by the determination of floc settlement velocity and image analysis. The frequency distribution of floc size was measured with a Malvern Mastersizer instrument. When the diatomite suspension was treated by MCPAM and CPAM, the residual diatomite turbidity was 16.28 NTU and 244.13 NTU, respectively. The maximum turbidity removal efficiency of MCPAM was about $99.65 \%$ under 1000 Gauss magnetic field, which was higher than that (94.75\%) of CPAM. The synergy of gravitational and magnetic fields for MCPAM promoted the formation of larger flocs with higher growth rates compared with CPAM. The effective density range of flocs in the MCPAM flocculation was increased to $10-252 \mathrm{~kg} \mathrm{~m}^{-3}$. The kinetic constants were calculated by monitoring the frequency of floc collisions. The increase of kinetic constant $(k)$ to $25.81 \times 10^{-11} \mathrm{~s}^{-1}$ suggested that interaction of contact and collision between magnetic flocs was sufficient. According to the evolution of the size and density of flocs under the synergy of gravitational and magnetic fields, the magnetic flocculation rate equation $\left((\mathrm{d} N / \mathrm{d} t)=-(1 / 9 \mu)\left[\left(\rho-\rho_{l}\right) g+\rho k_{m} H(\mathrm{~d} H / \mathrm{d} X)\right]\left(a_{i}^{2}-a_{j}^{2}-a_{i}^{2} e^{\left(9 \mu t / 2 a_{i}^{2} \rho\right)}+a_{j}^{2} e^{\left(9 \mu t / 2 a_{j}^{2} \rho\right)}\right)\left(a i+a_{j}\right)^{2}\right)$ was derived. The study of magnetic flocculation kinetics can provide theoretical support for magnetic flocculation and is critical for the analysis of solid-liquid separation processes.

\section{Introduction}

Flocculation is an important process in the water treatment system. And flocculants are a key in achieving high flocculation efficiency in the aggregation process. More attention has been paid to the improvement of flocculation performance of flocculants and development of water treatment technology.

Conventional organic flocculants, such as cationic polyacrylamide (CPAM), exhibit excellent flocculation performance in water treatment due to favorable adsorption and bridging. Nevertheless, the resuspension of flocs generated still remains unresolved. Magnetic flocculants provide good magnetic responsiveness, which is a combination of polymer compounds and magnetic substances. Magnetic flocculants have several advantages, fast flocs formation, shortened hydraulic retention time, and reduced interstitial water content of the flocs. In recent years, magnetic flocculants have been widely applied in microalgae harvesting, heavy metal removal, enhancing emulsification of water media, and tailings disposal, etc. [1-3]. Microalgae can be harvested by using magnetic nanocomposites, and $90 \%$ or above cell recovery can be achieved within 5 minutes [4-7]. In a short time, magnetic flocculants can remove heavy metal ions with low economic cost [8-11]. For instance, magnetic nanoparticle-supported layered double hydroxide nanocomposites (MLDO) could be an economical option for the removal system of $\mathrm{As}(\mathrm{V})$ and $\mathrm{Sb}(\mathrm{V})$ in water treatments 
considering competitive effect, maximum sorption capacity, and regeneration rate, due to their strong magnetic separability [12]. The magnetic flocculants have been used to treat emulsified oil wastewater with high treatment efficiency, easy recovery, and high emulsification performance [13-15].

Flocculation kinetics has evolved from a qualitative description to semiquantitative or quantitative model description. A variety of flocculation kinetics mathematical models in different conditions has been gradually established and continuously optimized. A new method for calculation of flocculation kinetics combining the Smoluchowski model with fractal theory was proposed by Yang [16]. In 2018, Wang et al. proposed a conceptual model to describe the microfloc growth under different shear rates, further revealing the reason for the different properties of flocs [17]. Runkana et al. proposed a model that can realistically describe the flocculation with a simple charge neutralization mechanism and track the evolution of floc size distribution [18]. Based on microparticle agglomeration characteristics, a flocculation concentration kinetic model was established by Gao et al. [19]. This model is conformed to the fractal characteristics of the particles and improved the applicability and accuracy of the concentration kinetic equation and the characteristic variables.

Previous studies on flocculation mainly include (1) flocculation performance and kinetics of new flocculants [20-22] and (2) influences of hydrodynamic conditions and intermolecular forces on flocculation kinetics $[23,24]$. Generally, the published research studies mainly focused on the flocculation behavior of conventional flocculants in the gravity field. However, the magnetic field strength is a very important parameter for the flocculation performance and kinetics of the magnetic flocculants. With the change of magnetic field strength, the flocculation speed and efficiency of flocculants constantly change. Accordingly, the relevant kinetic parameters also change. However, there are few reports available to establish the law of magnetic flocculation behavior under the cooperative action of gravity and magnetic fields. Therefore, it is very important to study the dynamics of magnetic flocculants in different magnetic fields.

In this work, the performance and flocculation kinetics of magnetic cationic polyacrylamide (MCPAM) under different magnetic field strengths were investigated and compared with cationic polyacrylamide (CPAM). The density of flocs was estimated by the determination of floc settlement velocity and image analysis. The frequency distribution of floc size was measured by the Malvern Mastersizer instrument. The kinetic constants for particle collisions and aggregation were calculated based on the bimolecular reaction model. Additionally, the flocculation rate equation of flocs was constructed under the synergistic action of magnetic and gravitational fields. The study of magnetic flocculation kinetics was completed.

\section{Materials and Methods}

2.1. Production of Magnetic Flocculant. The porous magnetic cationic polyacrylamide (MCPAM) microspheres were prepared by using $\mathrm{Fe}_{3} \mathrm{O}_{4}$, copolymerizing acrylamide (AM) and $\mathrm{N}, \mathrm{N}$-dimethylaminoethyl acrylate methyl chloride quarternary (DAC) via aqueous solution polymerization [25]. A $250 \mathrm{~mL}$ three-necked flask was placed in an oil bath. And $1.0 \mathrm{~g}$ of industrial gelatin, $4.13 \mathrm{~g}$ of $\mathrm{AM}$, and $0.65 \mathrm{~g}$ of DAC were dissolved in $30 \mathrm{~mL}$ deionized water. In a separate beaker, $0.5 \mathrm{~g}$ citric acid was dissolved in $5 \mathrm{~mL}$ deionized water and $2 \mathrm{~g}$ of $\mathrm{Fe}_{3} \mathrm{O}_{4}$ was put into it. Then, the mixture was poured into the three-necked flask. The redox initiator $0.072 \mathrm{~g}$ of sodium bisulfate and $0.072 \mathrm{~g}$ of ammonium persulfate were added into the flask, and the reaction mixture was stirred for 90 minutes at $80^{\circ} \mathrm{C}$. The extraction of the polymer was executed in ethanol, and the copolymer was dried in an oven at a constant temperature of $40^{\circ} \mathrm{C}$. The magnetic flocculant stock solution of $1.0 \mathrm{~g} \mathrm{~L}^{-1}$ was prepared by dissolving $0.1 \mathrm{~g}$ of magnetic flocculants in $100 \mathrm{~mL}$ of deionized water at room temperature $\left(25 \pm 1^{\circ} \mathrm{C}\right)$. The stock solution was used within two days of preparation.

2.2. Flocculation Experiments. In order to evaluate the performance of magnetic flocculants, a diatomite suspension was prepared. The diatomite suspension was prepared by dissolving $5.0 \mathrm{~g}$ of predried diatomite powder in $1.0 \mathrm{~L}$ of deionized water. The size of diatomite was distributed in $0-2 \mu \mathrm{m}, 2-4 \mu \mathrm{m}$, and $4-6 \mu \mathrm{m}$ which accounted for $42.21 \%, 48.36 \%$, and $9.23 \%$, respectively. The flocculation experiments were carried out in a conventional jar testing apparatus equipped with six flat paddle impellers and cylindrical jars containing $200 \mathrm{~mL}$ samples of diatomite suspension at room temperature of $25 \pm 1^{\circ} \mathrm{C}$. The mixture was rapidly stirred for 1 minute at $300 \mathrm{rpm}$ after adding $5 \mathrm{~mL}$ flocculant stock solution. The jar was placed inside a magnetic field created by a cubic neodymium permanent magnet with a magnetic field strength of 1000 Gauss, 500, and 0 Gauss, respectively. After magnetic flocculation, the supernatant was collected below the water level of $1-2 \mathrm{~cm}$, and then the transmittance was analyzed with a TU-1901 dual-beam ultraviolet/ visible spectrophotometer (wavelength $680 \mathrm{~nm}$, Beijing $\mathrm{Pu}$ Analysis General Instrument Co., Ltd., China). The wavelength of $680 \mathrm{~nm}$ was the maximum absorption wavelength of the tested sample, which was measured by ultraviolet/visible spectrophotometer (UV-2000, Shimadzu, Japan). All experiments had been replicated 3 times. In this experiment, the transmittance of supernatant treated by MCPAM under different magnetic field strengths was investigated and was compared with the performance of conventional cationic polyacrylamide (CPAM).

2.3. Analysis and Calculation. Floc size is one of the main parameters needed to describe a floc's structure. During the flocculation process, magnetic flocs can be formed after the addition of magnetic flocculants, and floc size will increase due to magnetic floc aggregation. A steady state of floc size occurs eventually due to the equilibrium between the aggregation and breakage of the magnetic flocs under the magnetic field. 
Malvern Mastersizer instrument (Mastersizer 3000, Malvern, UK) was used to monitor the dynamics of floc size as the flocculation proceeded. Jar tests were conducted in a similar fashion that the diatomite suspension was rapidly stirred for 1 minute at $300 \mathrm{rpm}$ after adding $5 \mathrm{~mL}$ magnetic flocculant stock solution. After stirring, the jar was placed on a cubic neodymium permanent magnet for gravity and magnetism settling. The suspension was measured by continuous recycling of water flowing through the sample cell of the instrument. A peristaltic pump (BT00-300, Longer Precision Pump, China) with a $5 \mathrm{~mm}$ internal diameter at a flow rate of $2.0 \mathrm{~L} \mathrm{~h}^{-1}$ was used to draw the suspension. The pump was located downstream of the instrument to prevent disturbing the flocs prior to measurement. The above conditions were maintained throughout all the tests, so that the results could be compared with each other. Floc size measurement was taken every 20 seconds in the measuring cell.

The floc density was another important parameter of flocculation. To further explore the kinetics of the magnetic flocculation, the evolution of floc density was analyzed under the applied magnetic field. There are many simple methods to measure the sizes of flocs. For instance, some commercial instruments use light scattering to determine floc size or a combination of photographic/video and image analysis. However, it is difficult to measure the floc density. Gregory [26] summarizes several different methods for measuring the density of flocs. Among them, the image method is widely used to calculate the density of flocs in many studies [27, 28]. In this work, the flocs density was measured by using the image method. The cross-sectional images of the flocs were captured by a charge-coupled device (CCD) camera (LC-30, Olympus, Japan) with a maximum resolution of 6250 pixels $/ \mathrm{mm}$. The maximum length and projected area of the flocs were measured.

The floc diameter $\left(d_{a}\right)$ is used and calculated using the following equation based on the projected area $\left(A_{i}\right)$ of the image:

$$
d_{a}=\left(\frac{4 A_{i}}{\pi}\right)^{0.5}
$$

The spherical porous flocs settle steadily in a medium by the following equation $[29,30]$ :

$$
\begin{aligned}
\frac{\rho_{a}-\rho_{L}}{\rho_{p}-\rho_{L}} & =1-\varepsilon \\
& =\frac{3 \rho_{L} \Omega C_{D}}{4 g\left(\rho_{p}-\rho_{L}\right) \cdot d_{a}} U_{a}^{2},
\end{aligned}
$$

where $\varepsilon$ is the floc overall porosity and $g$ is the gravitational acceleration.

The difference between floc density $\left(\rho_{a}\right)$ and fluid density $\left(\rho_{L}\right)$ is effective floc density $\left(\rho_{\text {Eff }}\right)$. During the experiment, the flocs' equivalent diameter $\left(d_{a}\right)$ can be measured. The separation velocity $\left(U_{a}\right)$ can be calculated using equation $U_{a}=(\Delta X / \Delta t)$, where $\Delta X$ is the flocs' settling distance and $\Delta t$ is the settling time. The other three unknown parameters in the above equation include the original floc density $\left(\rho_{p}\right)$, drag coefficient $\left(C_{D}\right)$, and correction factor
$(\Omega)$. These three parameters are calculated according to the previous studies [30, 31].

\section{Results and Discussion}

3.1. Flocculation Performance of MCPAM and CPAM in Different Magnetic Field Strength. The flocculation of diatomite suspension by MCPAM and CPAM under an external magnetic field was investigated. As shown in Figure 1 and Table 1, the transmittance of the supernatant was $90.32 \%$ at $40 \mathrm{~s}$ and slowly reached $95.14 \%$ at $100 \mathrm{~s}$ without an external magnetic field for MCPAM. When the applied magnetic field was increased to 500 Gauss, the transmittance of supernatant increased to $93.68 \%$ at $40 \mathrm{~s}$ and finally stabilized to $97.54 \%$ after 100 s. Under a 1000 Gauss of magnetic field, the transmittance of supernatant increased to $98.96 \%$ at $40 \mathrm{~s}$ after adding MCPAM and finally stabilized at $99.65 \%$ after $100 \mathrm{~s}$. The transmittance of supernatant was improved by $8.64 \%$ at $40 \mathrm{~s}$ as the applied magnetic field was from 0 to 1000. The results indicated that the efficiency of magnetic flocculants in high magnetic field intensity was higher than that in low magnetic field intensity. The strength of the magnetic field can affect the movement of magnetic flocs. The magnetic field can attract the magnetic flocs, and the flocs can also magnetically attract each other. As the magnetic field strength increases, the chance of collision and combination between the magnetic flocs also increases. The larger flocs are formed by the collision of magnetic flocs and then settle and separate quickly under the action of an external magnetic field. When MCPAM flocculates the suspension in the absence of a magnetic field, the transmittance of the supernatant will increase slowly because the flocs are only affected by the gravitational field. The collision efficiency between the flocs is not as high as those under magnetic field conditions. Therefore, the transmittance of supernatant may be different under different magnetic field strengths.

For CPAM, the transmittance of supernatant only increased to $85.62 \%$ at $40 \mathrm{~s}$ and stabilized at $94.75 \%$ after $100 \mathrm{~s}$ under a 1000 Gauss of magnetic field (Figure 1 and Table 1). The flocculation efficiency of CPAM was much lower than that of MCPAM. The results indicated that the magnetic flocculant could improve the treatment efficiency and shorten the flocculation time. There were two main reasons for this phenomenon. Firstly, MCPAM microspheres make the negatively charged suspended particles unstable through the bridging mechanism. According to reports, magnetic flocculant has a high molecular weight, and the macromolecular chains form molecular bridges via hydrogen bonds to adsorb suspended particles [32]. Secondly, MCPAM microspheres have a core-shell structure, which is $\mathrm{Fe}_{3} \mathrm{O}_{4}$ nanoparticles coated with polyacrylamide, resulting in good magnetic responsiveness. The resulting magnetic flocs have a faster separation speed under the synergistic action of the gravitational and magnetic fields; however, the floc formed by the conventional flocculant and separate suspension were only attributed to gravity. ANOVA showed that the population mean of the time and flocculant type were significantly different as listed in Table 2. $p$ value of all 


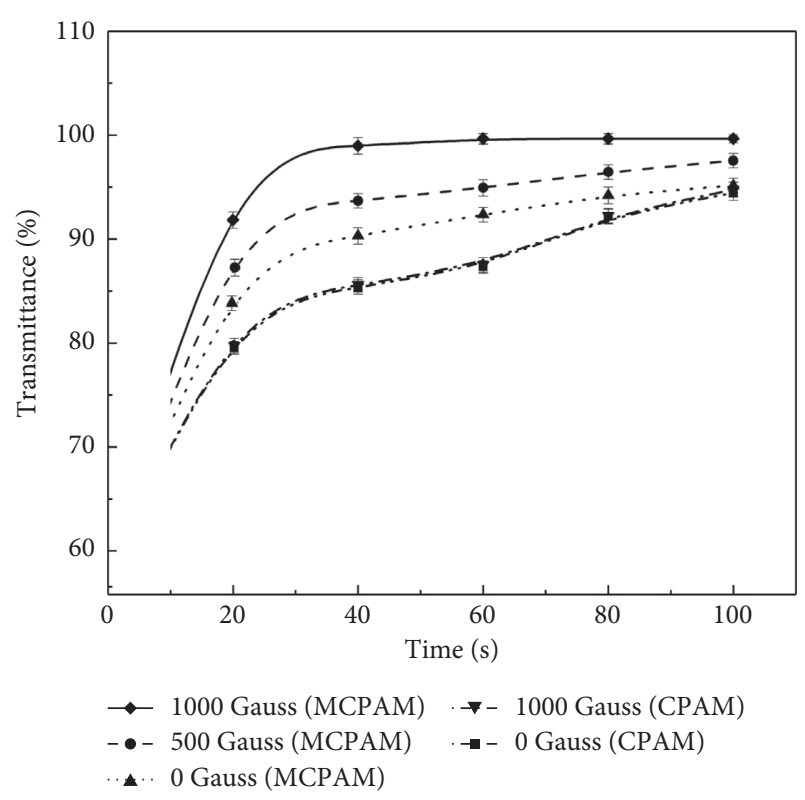

FIgURE 1: Changes of supernatant transmittance in the diatomite suspension treatment by the MCPAM and CPAM under different magnetic field strengths.

TABle 1: The flocculation rate constant for diatomite suspensions with various flocculants.

\begin{tabular}{lcccc}
\hline Flocculant & \multicolumn{2}{c}{ MCPAM } & \multicolumn{2}{c}{ CPAM } \\
\hline Electric filed strength (Gauss) & 1000 & 500 & 0 & 1000 \\
Transmittance $(\%, t=40 \mathrm{~s})$ & 98.96 & 93.68 & 90.32 & 85.62 \\
Transmittance $(\%, t=100 \mathrm{~s})$ & 99.65 & 97.54 & 95.14 & 94.75 \\
Effective density range $\left(\mathrm{kgm}^{-3}\right)$ & $10-252$ & - & - & $7-226$ \\
Floc size range $(\mu \mathrm{m})$ & $48-724$ & - & - & $40-549$ \\
$k\left(\times 10^{-11} \mathrm{~s}^{-1}\right)$ & 6.16 & - & - & 25.81 \\
$R^{2}$ & 0.97 & - & - & 0.90 \\
\hline
\end{tabular}

TABle 2: Two-way ANOVA on different indicators in the flocculation process.

\begin{tabular}{lccc}
\hline & Sum of squares & Mean square & $p$ \\
\hline Time & 157.737 & 39.434 & 0.000 \\
Type of flocculants & 832.555 & 832.555 & 0.000 \\
\hline
\end{tabular}

indicators was less than 0.05 . These results indicate that the interaction between time and type of flocculant was significant.

3.2. Evolution of the Floc Size. For the diatomite suspension treated by MCPAM and CPAM of $1 \mathrm{~g} \mathrm{~L}^{-1}$, the changes of floc size are listed in Figure 2 to describe the evolution of the flocculation process. The magnetic field was 1000 Gauss. In the MCPAM reactor, the particular percentage of $150-200 \mu \mathrm{m}$ (the dominant floc size) was $26 \%$ at $20 \mathrm{~s}$ and gradually reduced to $10 \%$ at $100 \mathrm{~s}$ (Figure $2(\mathrm{a})$ ). The specific percentage of $250-300 \mu \mathrm{m}$ was only $5 \%$ at $20 \mathrm{~s}$ and increased to $18 \%$ at $100 \mathrm{~s}$. And $250-300 \mu \mathrm{m}$ became the predominant floc size at the end of magnetic flocculation. The same appearance was obtained for $300-500 \mu \mathrm{m}$, which implied that the magnetic flocs were effectively agglomerated in the magnetic flocculation. The specific percentage of $300-350 \mu \mathrm{m}$ was $5 \%$ at $20 \mathrm{~s}$ and increased to $15 \%$ at $100 \mathrm{~s}$. The specific percentage of $350-400 \mu \mathrm{m}$ was only $3 \%$ at $20 \mathrm{~s}$ and increased to $8 \%$ at $100 \mathrm{~s}$. The particular percentage of $450-500 \mu \mathrm{m}$ was $2 \%$ at $60 \mathrm{~s}$ and gradually increased to $6 \%$ at $100 \mathrm{~s}$. In the CPAM reactor, the particular percentage of $100-150 \mu \mathrm{m}$ (predominant floc size) was $44 \%$ at $20 \mathrm{~s}$ and dropped to $19 \%$ at $100 \mathrm{~s}$ (Figure 2(b)). The particular percentage of $150-200$ (main floc size) $\mu \mathrm{m}$ was $24 \%$ at the end of the CPAM flocculation. At the same flocculation time, the CPAM flocs of 300-500 $\mu \mathrm{m}$ were less than that of MCPAM. This indicates that the MCPAM flocs have a better cohesive force than the CPAM flocs. According to previous studies, magnetic fields can attract magnetic flocs, and the flocs also magnetically attract each other $[33,34]$. Consistent with this study, the size proportion of magnetic flocs was larger than that of conventional flocs.

The combination of charge neutralization and surface bridging flocculation is pertinent to increasing flocs size and growth rate by magnetic flocculation [33]. In addition, magnetic flocs themselves are magnetic, making them attractive enough to absorb charged particles. The magnetic fields lead to an enhancement in magnetic flocs collision efficiency, promoting the formation of flocs with larger sizes and higher growth rate. Consequently, MCPAM produced flocs with larger size depending on its stronger charge neutralization and magnetic attraction ability than the conventional flocculants. In the early stages of the process, aggregation was the dominant mechanism. After the flocs grew, fragmentation competed with aggregation. Finally, a dynamic balance between aggregation and fragmented was established. As depicted in Figure 2, the size distribution of flocculation was similar at $80 \mathrm{~s}$ and $100 \mathrm{~s}$, indicating that the floc size distribution does not change considerably if the flocculation time was over $80 \mathrm{~s}$.

3.3. Evolution of the Floc Density. Under 1000 Gauss magnetic field, the diatomite suspension was treated with MCPAM and CPAM of $1 \mathrm{~g} \mathrm{~L}^{-1}$. The projected area of the flocs was determined via image analysis after magnetic flocculation, and diameters of about 200 flocs were measured for each experiment. Figure 3 shows the photographs of MCPAM and CPAM flocs at 1000 Gauss magnetic field. The magic MCPAM flocs were much larger than the conventional flocs.

After measuring the projected area of the flocs, the separation velocity $\left(U_{a}\right)$ is calculated. $U_{a}$ of the magnetic flocs exhibits a power law relationship with floc diameter as $U_{a} \propto d_{a}^{0.84}$ at $t=20 \mathrm{~s}$ and $U_{a} \propto d_{a}^{0.87}$ at $t=100 \mathrm{~s}$. $U_{a}$ of the conventional flocs is $U_{a} \propto d_{a}^{0.82}$ at $t=20 \mathrm{~s}$ and $U_{a} \propto d_{a}^{0.85}$ at $t=100 \mathrm{~s}$. The results indicated that the magnetic field strength notably accelerated the separation process. The separation occurs owing to diverse forces acting on the magnetic flocs under an external field. These forces include magnetic attraction, gravitational, and interparticle forces 


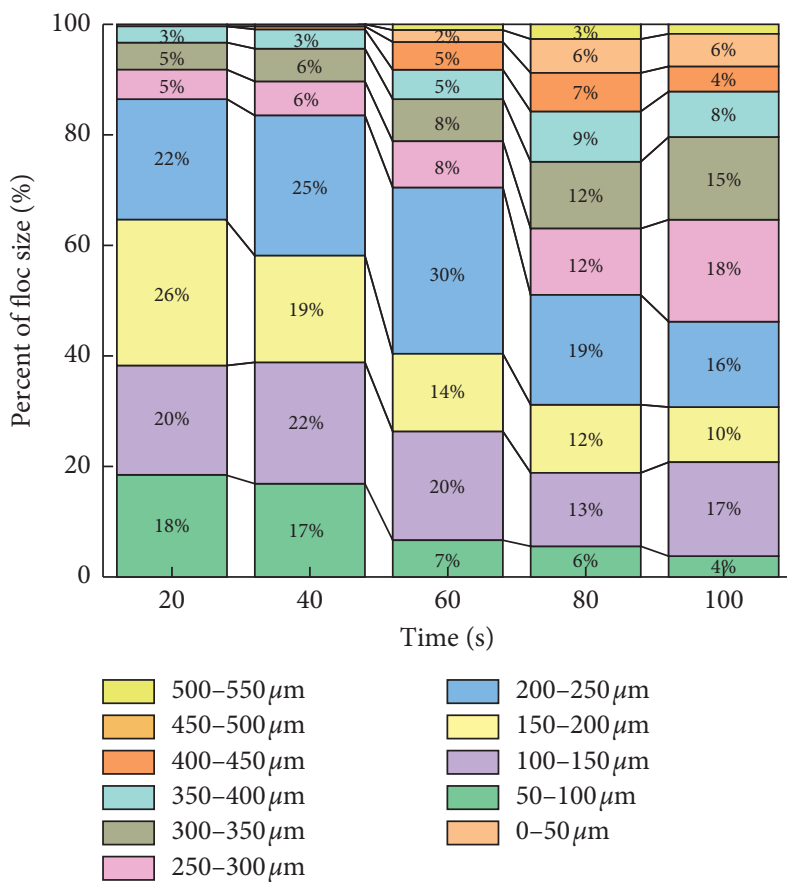

(a)

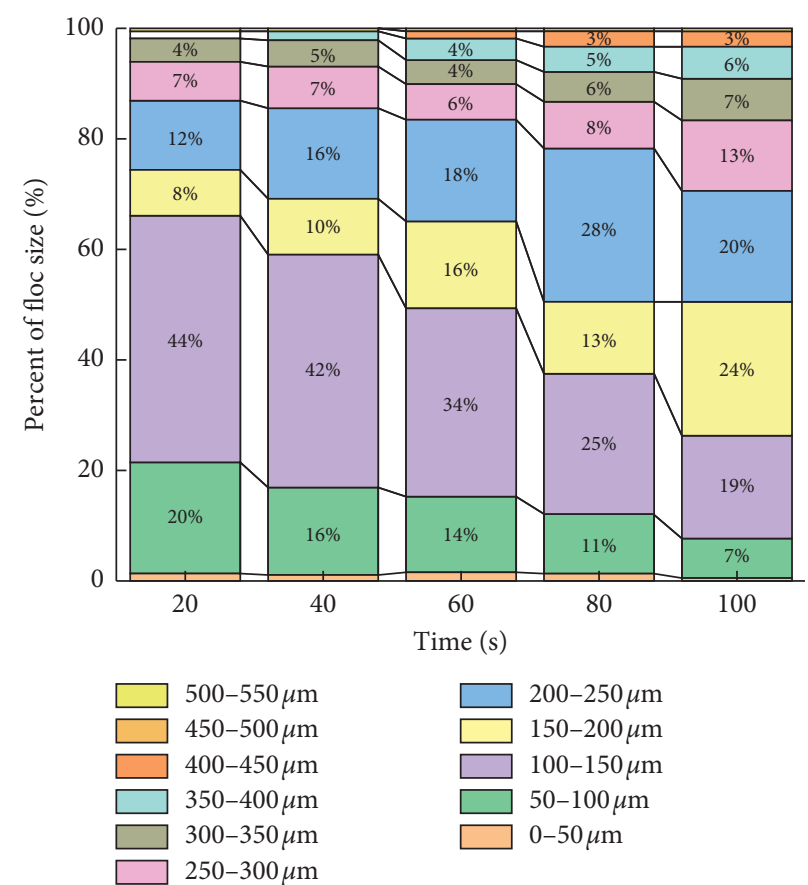

(b)

FIgURE 2: The floc size distribution for the (a)MCPAM flocculation and (b)CPAM flocculation.

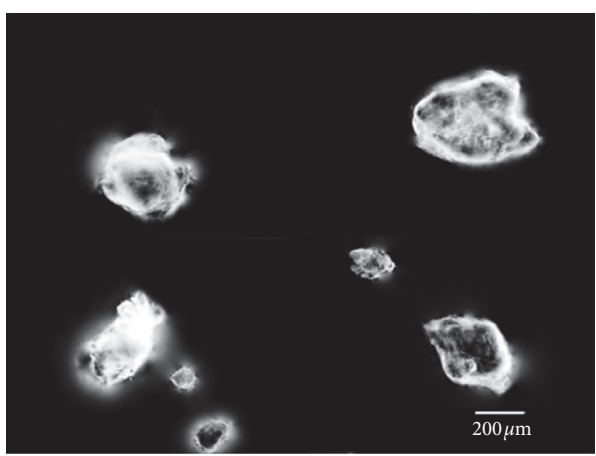

(a)

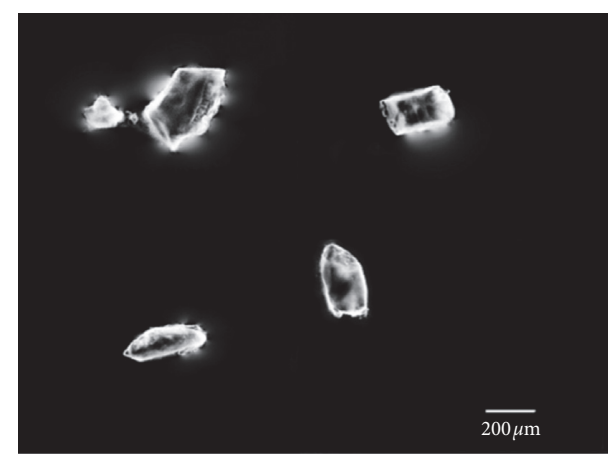

(b)

Figure 3: Images of the (a)MCPAM flocs and (b)CPAM flocs.

such as the van der Waals forces, hydrodynamic resistance, and magnetic dipole attraction forces [33]. The magnetic force overcomes other competitiveness and yields a field that is an order of magnitude stronger than ordinary gravity sedimentation.

The size of the primary particle (diatomite particle before destabilization) was $0.54 \mu \mathrm{m}$, as measured with a Malvern Mastersizer (Mastersizer 3000, Malvern, UK). The density of the primary particle was $2280 \mathrm{~kg} \mathrm{~m}^{-3}$ (reported by the supplier). Destabilization of dispersed primary particles was done by a coagulation step and formation of microflocs, hereafter called primary flocs. The primary floc size was $2.5 \mu \mathrm{m}$. These data were used to calculate the primary floc density $\left(\rho_{p}\right)$ $\left(\rho_{p}=1497 \mathrm{~kg} \cdot \mathrm{m}^{-3}\right)$, which was used in equation (2) to determine effective floc density $\left(\rho_{\text {Eff }}\right)$. The relationship between the effective floc density and diameter is shown in Figure 4 . The $\rho_{\text {Eff }}$ value decreases with increasing floc diameter. The density of MCPAM flocs was always higher than that of CPAM flocs. This is a typical property of magnetic flocs, and the related results are in agreement with previous research findings [30]. After flocculation, $\rho_{\text {Eff }}$ ranges of CPAM and MCPAM flocs were $7-226 \mathrm{~kg}$ $\mathrm{m}^{-3}$ and $10-252 \mathrm{~kg} \mathrm{~m}^{-3}$, respectively (Table 1 ). MCPAM can flocculate the suspension to form flocs with higher effective density in a short time under the synergistic effect of gravitational and magnetic fields. The magnetic flocs were exposed to the applied magnetic field, causing the magnetic flocs to aggregate when the magnetic attraction between the induced or permanent magnetic moments 


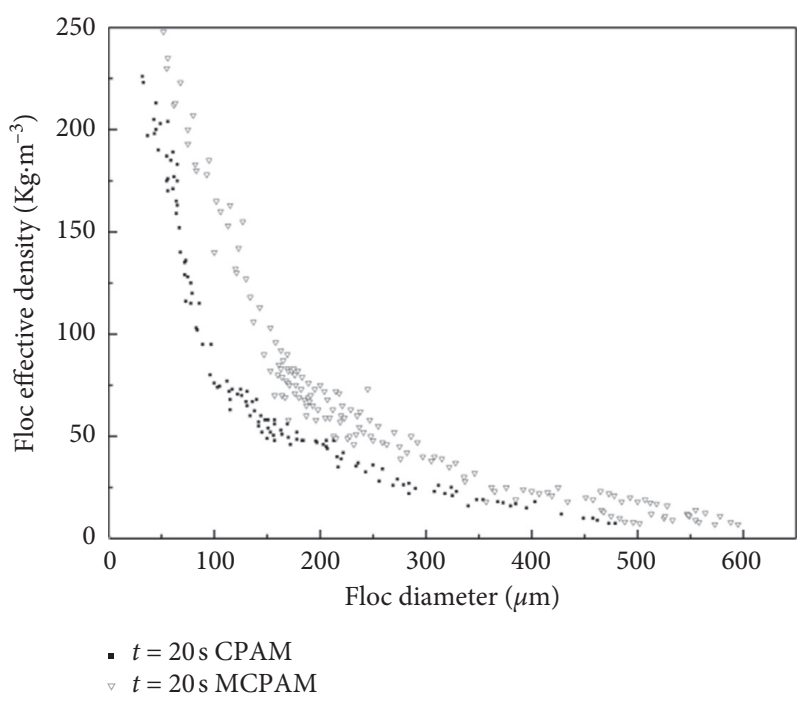

(a)

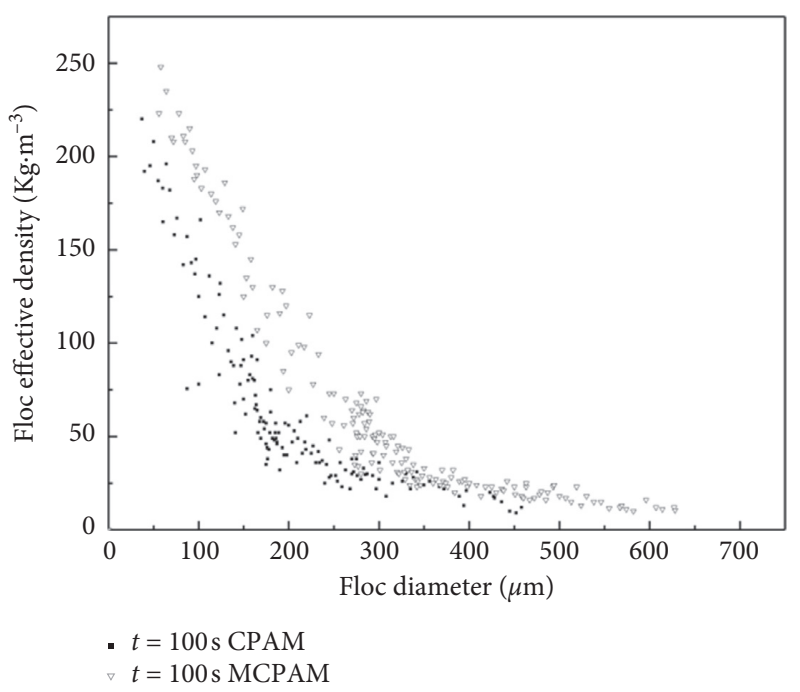

(b)

FIgURE 4: Relationship between floc diameter and effective density at (a)20 seconds and (b)100 seconds in the MCPAM and CPAM flocculations.

was sufficient to exceed the stabilizing force of electrochemical origin. Therefore, the magnetic flocs attract each other due to their good magnetic responsiveness and eventually form tighter and more condensed flocs with fewer pores.

3.4. Frequency of Floc Collisions. The study of flocculation kinetics is very important for understanding the flocculation mechanism and the process control of solid-liquid system separations. In this work, the MCPAM and CPAM were selected as representative flocculants for kinetic studies of conventional and magnetic flocculants. Previous studies suggest that the order of flocculation was mostly bimolecular as expressed in the following equation [33,34]:

$$
\left(\frac{N_{0}}{N_{t}}\right)^{1 / 2}=1+\frac{1}{2} k N_{0} t
$$

where $N_{t}$ is the concentration of diatomite particles with respect to time $(t), N_{0}$ is the initial concentration of diatomite particles, $k$ is the kinetic constant of particle collisions and aggregation. $N_{0}$ value for diatomite of known weight is calculated by considering the particle diameter $(0.54 \mu \mathrm{m})$ and diatomite density $\left(2.28 \mathrm{~g} \cdot \mathrm{cm}^{-3}\right) . N_{0}$ is the fixed value for all diatomite suspensions with the same weight in this experiment. In addition, the relationship between the concentration of diatomite suspension and transmittance of supernatant is shown in the following equation:

$$
\frac{N_{0}}{N_{t}}=\frac{\left(100-T_{0}\right)}{\left(100-T_{t}\right)}
$$

where $T_{0}(\%)$ is the transmittance of the initial diatomite suspension and $T_{t}(\%)$ is the transmittance of the supernatant at time $t(\mathrm{~s})$. Therefore, the functional relationship between $N_{0} / N_{t}$ and $t$ can be linearly fitted based on the

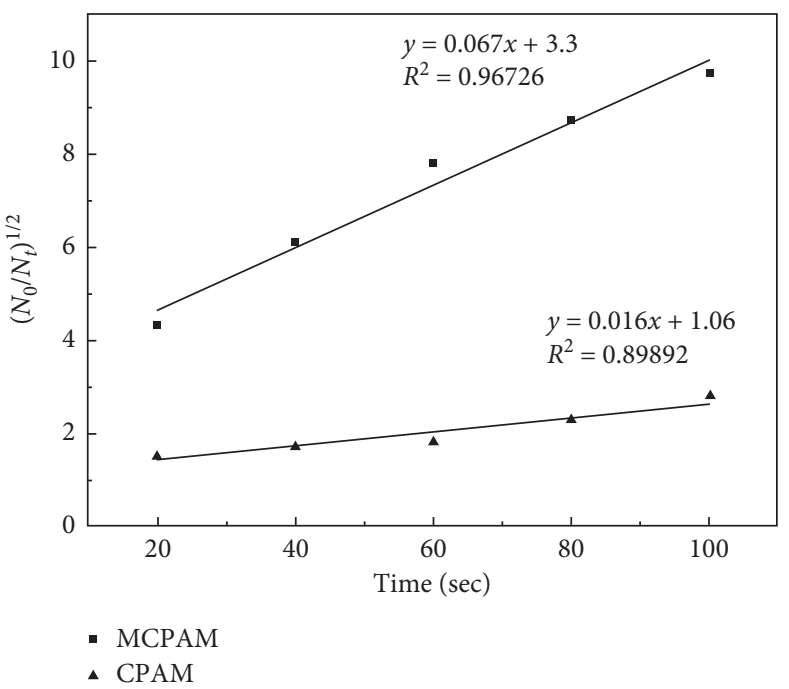

FIgURE 5: $\left(N_{0} / N_{t}\right)^{1 / 2}$ as a function of separation time in the MCPAM and CPAM flocculations.

original data as shown in Figure 1. The fitting results are presented in Figure 5.

The kinetic constants $(k)$ can be calculated by using the slope of the fitting curve between $\left(N_{0} / N_{t}\right)^{1 / 2}$ and flocculation time $t$ and are listed in Table 1 . The rate constant $k$ of the magnetic flocculant MCPAM was higher than that of the conventional flocculant CPAM under the external magnetic field. The maximum $k$ value was $25.81 \times 10^{-11} \mathrm{~s}^{-1}$ for MCPAM flocculation under 1000 Gauss magnetic field. This suggested that the most efficient collision occurred between magnetic flocs under the synergy of gravity and magnetic fields. This may be due to charge neutralization and magnetic attraction that jointly control the magnetic flocculation process, which plays a key role in producing larger and stronger flocs $[35,36]$. The flocs 
settling at a higher speed will collide with those settling at a lower speed. Especially, under the synergistic effect of magnetic and gravitational fields, the collision and aggregation of flocs were accelerated. Therefore, the flocs mass rapidly increased. This increased the separation speed of the flocs and made it collide with other flocs and aggregate further. However, the flocs produced by conventional flocculants do not have magnetic responsiveness, so the $k$ value is low.

Under the synergistic effect of magnetic field and gravitational field, the collision frequency and collision efficiency between magnetic flocs were enhanced, and the larger flocs with higher growth rate were formed in a short time, resulting in higher transmittance of supernatant. This is consistent with the previous performance analysis.

3.5. Derivation of the Flocculation Rate Equation. Based on the above results, the collision rate equation was developed by considering the synergy of magnetic and gravitational fields.

When the direction of the magnetic field coincides with that of the gravitational field, the gravity $\left(F_{g}\right)$ in the medium is shown in equation (5) assuming the volume of a spherical particle is $V$ and diameter is $d_{a}$ :

$$
F_{g}=\frac{\pi}{6} d_{a}^{3} \rho g .
$$

The magnetic floc is subjected to magnetic field force $\left(F_{z}\right)$, buoyancy $\left(F_{f}\right)$, and fluid resistance $\left(F_{p}\right)$, respectively:

$$
\begin{aligned}
& F_{z}=\frac{\pi}{6} d_{a}^{3} \rho k_{m} H \frac{\mathrm{d} H}{\mathrm{~d} X}, \\
& F_{f}=\frac{\pi}{6} d_{a}^{3} \rho_{l} g, \\
& F_{p}=3 \pi \mu v d_{a},
\end{aligned}
$$

where $k_{m}$ is the magnetic susceptibility, $H$ is the magnetic field strength, $d H / d X$ is the magnetic field intensity gradient, $\rho$ is the particle density, $\rho_{l}$ is the liquid density, $\mu$ is the dynamic viscosity coefficient of the liquid, and $v$ is the particle sedimentation velocity. When the gravitational and magnetic fields were in the same direction, the following results can be obtained:

$$
\begin{aligned}
& m \frac{\mathrm{d} v}{\mathrm{~d} t}=F_{z}+F_{g}-F_{f}-F_{p}, \\
& m \frac{\mathrm{d} v}{\mathrm{~d} t}=\frac{\pi}{6}(\rho-\rho l) d_{a}^{3} g+\frac{\pi}{6} d_{a}^{3} \rho K m H \frac{\mathrm{d} H}{\mathrm{~d} X}-3 \pi \mu v d_{a} .
\end{aligned}
$$

By integrating equation (8), the following results can be obtained:

$$
v=\frac{d_{a}^{2}\left[\left(\rho-\rho_{l}\right) g+\rho k_{m} H(\mathrm{~d} H / \mathrm{d} X)\right]}{18 \mu}\left(1-e^{\left(18 \mu t / \rho d_{a}^{2}\right)}\right) .
$$

When the flocs are only subjected to gravitational field, Strokes' theorem states that the separation velocity of flocs in water can be expressed as follows [29]:

$$
v_{g}=\frac{\left(\rho-\rho_{l}\right) g d_{a}^{2}}{18 \mu} .
$$

After the destabilization of the suspension, the separation speeds of particles were different depending on the sizes and densities of the particles under the synergistic effect of gravity and magnetic fields. The separation speed difference also led to the collision of particles. Here, we consider two kinds of particles with radii $a_{i}$ and $a_{j}$, respectively. According to equation (9), if two kinds of particles with $a_{i}>a_{j}$ have the same density, the difference in the separation speed between them can be expressed as follows:

$$
\begin{aligned}
v_{i}-v_{j}= & \frac{2\left[\left(\rho-\rho_{l}\right) g+\rho k_{m} H(\mathrm{~d} H / \mathrm{d} X)\right]}{9 \mu} \\
& \cdot\left(a_{i}^{2}-a_{j}^{2}-a_{i}^{2} e^{\left(9 \mu t / 2 a_{i}^{2} \rho\right)}+a_{j}^{2} e^{\left(9 \mu t / 2 a_{j}^{2} \rho\right)}\right),
\end{aligned}
$$

where $v_{i}$ and $v_{j}$ are the velocities of particles $i$ and $j$, respectively.

This equation considers that particle $i$ with a high separation speed catches up with particle $j$ and then collides and aggregates. The collision radius centered on particle $j$ was $R=a_{i}+a_{j}$, so there was a plane $\pi R^{2}$ centered on particle $j$. Collision and binding will occur when particle $i$ enters this plane. The particle weight of the cylinder section with radius $R$ was expressed as follows:

$$
N_{i} \frac{\mathrm{d} v_{l}}{\mathrm{~d} t}=N_{i} \pi R^{2}\left(v_{i}-v_{j}\right),
$$

where $\mathrm{d} v_{l} / \mathrm{d} t$ was the volume flow rate, that is, the flow rate. Since the number of particles $j$ per unit volume was $N_{j}$, the collision rate can be expressed as follows:

$$
N_{i} N_{j} \frac{\mathrm{d} v_{l}}{\mathrm{~d} t}=N_{i} N_{j}\left(a_{i}+a_{j}\right)^{2}\left(v_{i}-v_{j}\right) .
$$

Substituting equation (11) into equation (13) can obtain the collision rate of particles under the synergistic effects of magnetic and gravitational fields:

$$
\begin{aligned}
N_{i} N_{j} \frac{\mathrm{d} v_{l}}{\mathrm{~d} t}= & N_{i} N_{j} \frac{2\left[\left(\rho-\rho_{l}\right) g+\rho k_{m} H(\mathrm{~d} H / \mathrm{d} X)\right]}{9 \mu} \\
& \cdot\left(a_{i}^{2}-a_{j}^{2}-a_{i}^{2} e^{\left(9 \mu t / 2 a_{i}^{2} \rho\right)}+a_{j}^{2} e^{\left(9 \mu t / 2 a_{j}^{2} \rho\right)}\right)\left(a_{i}+a_{j}\right)^{2} .
\end{aligned}
$$

Therefore, the magnetic flocculation rate equation is expressed as follows:

$$
\begin{aligned}
\frac{\mathrm{d} N}{\mathrm{~d} t}= & -\frac{1}{9 \mu}\left[\left(\rho-\rho_{l}\right) g+\rho k_{m} H \frac{\mathrm{d} H}{\mathrm{~d} X}\right] \\
& \cdot\left(a_{i}^{2}-a_{j}^{2}-a_{i}^{2} e^{\left(9 \mu t / 2 a_{i}^{2} \rho\right)}+a_{j}^{2} e^{\left(9 \mu t / 2 a_{j}^{2} \rho\right)}\right)\left(a_{i}+a_{j}\right)^{2} .
\end{aligned}
$$




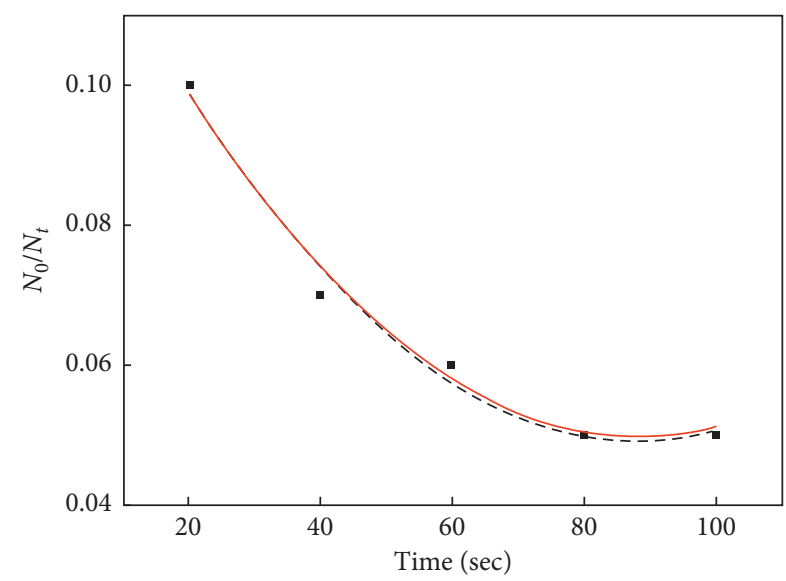

- Experimental data

- - - Fitting curve of experimental data

Theoretic curve

FIGURE 6: Kinetic curves of flocculation processes for diatomite suspension with MCPAM.

Equation (15) is a modified formula, which can describe the flocculation rate of flocs under the synergy of magnetic and gravitational fields. In Figure 6, the derived equation (15) was verified by the experimental data. The results show that the fitting curve of experimental data almost coincides with the curve of derived equation (15). It indicates that the modified flocculation rate equation is in a high agreement with the actual flocculation processes. Magnetic flocculants offer good magnetic responsiveness and can efficiently achieve solid-liquid separation under the magnetic field. Magnetic flocculants can shorten the hydraulic retention time, so as to achieve efficient and rapid flocculation. In order to further understand the specific process of particle flocculation and give details of the role of magnetic flocculation, it is necessary to study the dynamics of magnetic flocculation. This study can provide optimal conditions for magnetic flocculation to guide the engineering practice. With the application of magnetic flocculants, the development of an efficient magnetic flocculation reactor may be an important link in the development of flocculation technology.

\section{Conclusions}

The suspension removal efficiency of MCPAM was stable at 99.65\% under 1000 Gauss magnetic field, which was higher than $94.75 \%$ of CPAM. The synergy of gravitational and magnetic fields can promote the size of magnetic floc growth performance during the flocculation of diatomite suspension. The floc density increased to $10-252 \mathrm{~kg} \cdot \mathrm{m}^{-3}$, and the flocs became tighter and more condensed. The magnetic flocculant has a better flocculation performance than the conventional one. The kinetic constant $k$ in the MCPAM flocculation was increased to $25.81 \times 10^{-11} \mathrm{~s}^{-1}$. The magnetic flocs attracted each other under the synergistic effects of gravity and magnetic fields, thereby increasing the collision frequency and efficiency between the magnetic flocs. The magnetic flocculation rate equation $\left((\mathrm{d} N / \mathrm{d} t)=-(1 / 9 \mu)\left[\left(\rho-\rho_{l}\right) g+\rho k_{m} H(\mathrm{~d} H / \mathrm{d} X)\right]\left(a_{i}^{2}-a_{j}^{2}-\right.\right.$ $\left.\left.a_{i}^{2} e^{\left(9 \mu t / 2 a_{i}^{2} \rho\right)}+a_{j}^{2} e^{\left(9 \mu t / 2 a_{j}^{2} \rho\right)}\right)\left(a_{i}+a_{j}\right)^{2}\right)$ of the magnetic flocs under the synergy of the gravity and the magnetic fields was obtained. The magnetic field strength was added as a variable in the modified flocculation rate equation, which could provide an important measuring method for magnetic flocculation.

\section{Data Availability}

The data used to support the findings of this study are available from the corresponding author upon request.

\section{Conflicts of Interest}

The authors declare no conflicts of interest.

\section{Acknowledgments}

This work was supported by the Natural Science Foundation of Shandong Province, China (Grant ZR2014EEM044).

\section{Supplementary Materials}

The supplementary material is a graphical abstract, which describes the working principle of magnetic flocculant. In the graphical abstract, the magnetic flocculant shows an excellent turbidity removal efficiency, compared to the conventional flocculant. The magnetic flocculation rate equation of the magnetic flocculant under the synergy of the gravity and the magnetic fields is also shown in the graphical abstract. (Supplementary Materials)

\section{References}

[1] S. Li, X. M. Wang, and Q. L. Zhang, "Dynamic experiments on flocculation and sedimentation of argillized ultrafine tailings using fly-ash-based magnetic coagulant," Transactions of Nonferrous Metals Society of China, vol. 26, no. 7, pp. 19751984, 2016.

[2] S. Li and X. M. Wang, "Fly-ash-based magnetic coagulant for rapid sedimentation of electronegative slimes and ultrafine tailings," Powder Technology, vol. 303, pp. 20-26, 2016.

[3] J. Li, J. Zhu, S. Qiao et al., "Processing of coal fly ash magnetic spheres for clay water flocculation," International Journal of Mineral Processing, vol. 169, pp. 162-167, 2017.

[4] T. Wang, W. L. Yang, Y. Hong, and Y. L. Hou, "Magnetic nanoparticles grafted with amino-riched dendrimer as magnetic flocculant for efficient harvesting of oleaginous microalgae," Chemical Engineering Journal, vol. 297, pp. 304-314, 2016.

[5] Y. Zhao, W. Liang, L. Liu, F. Li, Q. Fan, and X. Sun, "Harvesting Chlorella vulgaris by magnetic flocculation using $\mathrm{Fe}_{3} \mathrm{O}_{4}$ coating with polyaluminium chloride and polyacrylamide," Bioresource Technology, vol. 198, pp. 789-796, 2015.

[6] K. Lee, J. G. Na, J. Y. Seo et al., "Magnetic-nanoflocculantassisted water-nonpolar solvent interface sieve for microalgae harvesting," ACS Applied Materials \& Interfaces, vol. 7, no. 33, pp. 18336-18343, 2015. 
[7] J. Y. Seo, K. Lee, R. Praveenkumar et al., "Tri-functionality of $\mathrm{Fe}_{3} \mathrm{O}_{4}$-embedded carbon microparticles in microalgae harvesting," Chemical Engineering Journal, vol. 280, pp. 206-214, 2015.

[8] B. Liu, X. Chen, H. Zheng et al., "Rapid and efficient removal of heavy metal and cationic dye by carboxylate-rich magnetic chitosan flocculants: role of ionic groups," Carbohydrate Polymers, vol. 181, pp. 327-336, 2018.

[9] N. Gu, C. Wang, J. Zhang, and T. Shen, "Synthesis and characterization of magnetic poly (Acrylamide-co-maleic anhydride) grafted gelatin as a novel heavy metal ions wastewater treatment agent," Desalination and Water Treatment, vol. 116, pp. 170-178, 2018.

[10] P. Yan, M. Ye, Z. Guan, S. Sun, Y. Guo, and J. Liu, "Synthesis of magnetic dithiocarbamate chelating resin and its absorption behavior for ethylenediaminetetraacetic acid copper," Process Safety and Environmental Protection, vol. 123, pp. 130-139, 2019.

[11] X. Song, L. Li, L. Zhou, and P. Chen, "Magnetic thiolated/ quaternized-chitosan composites design and application for various heavy metal ions removal, including cation and anion," Chemical Engineering Research and Design, vol. 136, pp. 581-592, 2018.

[12] S. H. Lee, H. Choi, and K. W. Kim, "Removal of As (V) and Sb $(\mathrm{V})$ in water using magnetic nanoparticle-supported layered double hydroxide nanocomposites," Journal of Geochemical Exploration, vol. 184, pp. 247-254, 2018.

[13] T. Lü, S. Zhang, D. Qi, D. Zhang, and H. Zhao, "Enhanced demulsification from aqueous media by using magnetic chitosan-based flocculant," Journal of Colloid and Interface Science, vol. 518, pp. 76-83, 2018.

[14] T. Lü, Y. Chen, D. Qi, Z. Cao, D. Zhang, and H. Zhao, "Treatment of emulsified oil wastewaters by using chitosan grafted magnetic nanoparticles," Journal of Alloys and Compounds, vol. 518, pp. 76-83, 2017.

[15] J. Tang, J. Wang, H. Jia et al., "The investigation on $\mathrm{Fe}_{3} \mathrm{O}_{4}$ magnetic flocculation for high efficiency treatment of oily micro-polluted water," Journal of Environmental Management, vol. 244, pp. 399-407, 2019.

[16] Z. Yang, H. Yang, Z. Jiang et al., "A new method for calculation of flocculation kinetics combining Smoluchowski model with fractal theory," Colloids and Surfaces A: Physicochemical and Engineering Aspects, vol. 423, pp. 11-19, 2013.

[17] Z. Wang, J. Nan, X. Ji, and Y. Yang, "Effect of the microflocculation stage on the flocculation/sedimentation process: the role of shear rate," Science of The Total Environment, vol. 633, pp. 1183-1191, 2018.

[18] V. Runkana, P. Somasundaran, and P. C. Kapur, "Mathematical modeling of polymer-induced flocculation by charge neutralization," Journal of Colloid and Interface Science, vol. 270, no. 2, pp. 347-358, 2004.

[19] R. Gao, K. Zhou, J. Zhang, H. Guo, and Q. Ren, "Research on the dynamic characteristics in the flocculation process of mineral processing tailings," IEEE Access, vol. 7, 2019.

[20] Z. Yang, X. Liu, B. Gao et al., "Flocculation kinetics and floc characteristics of dye wastewater by polyferric chloride-polyepichlorohydrin-dimethylamine composite flocculant," Separation and Purification Technology, vol. 118, pp. 583-590, 2013.

[21] P. Peng and G. Garnier, "Effect of cationic polyacrylamide on precipitated calcium carbonate flocculation: kinetics, charge density and ionic strength," Colloids and Surfaces A: Physicochemical and Engineering Aspects, vol. 408, pp. 32-39, 2012.
[22] A. Y. Zahrim, Z. D. Dexter, C. G. Joseph, and N. Hilal, "Effective coagulation-flocculation treatment of highly polluted palm oil mill biogas plant wastewater using dual coagulants: decolourisation, kinetics and phytotoxicity studies," Journal of Water Process Engineering, vol. 16, pp. 258-269, 2017.

[23] R. Meszaros, S. Barany, and I. Solomentseva, "Effect of hydrodynamic conditions on the kinetics of bentonite suspension flocculation by cationic polyelectrolytes and the strength of formed flocs," Colloid Journal, vol. 72, no. 3, pp. 409-416, 2010.

[24] L. Feng, Y. Adachi, and A. Kobayashi, "Kinetics of Brownian flocculation of polystyrene latex by cationic polyelectrolyte as a function of ionic strength," Colloids and Surfaces A: Physicochemical and Engineering Aspects, vol. 440, pp. 155160, 2014

[25] C. Wang, Y. Wang, Z. Ouyang, T. Shen, and X. Wang, "Preparation and characterization of polymer-coated $\mathrm{Fe}_{3} \mathrm{O}_{4}$ magnetic flocculant," Separation Science and Technology, vol. 53, no. 5, pp. 814-822, 2018.

[26] J. Gregory, “The density of particle aggregates," Water Science and Technology, vol. 36, no. 4, 1997.

[27] M. S. Nasser and A. E. James, "Effect of polyacrylamide polymers on floc size and rheological behaviour of kaolinite suspensions," Colloids and Surfaces A: Physicochemical and Engineering Aspects, vol. 301, no. 1-3, pp. 311-322, 2007.

[28] H. Huang, "Porosity-size relationship of drilling mud flocs: fractal structure," Clays and Clay Minerals, vol. 41, no. 3, pp. 373-379, 1993.

[29] C. P. Johnson, X. Li, and B. E. Logan, "Settling velocities of fractal aggregates," Environmental Science \& Technology, vol. 30, no. 6, pp. 1911-1918, 1996.

[30] D. J. Lee, G. W. Chen, Y. C. Liao, and C. C. Hsieh, "On the free-settling test for estimating activated sludge floc density," Water Research, vol. 30, no. 5, pp. 541-550, 1996.

[31] F. Vaezi, G. R. S. Sanders, and J. H. Masliyah, "Flocculation kinetics and aggregate structure of kaolinite mixtures in laminar tube flow," Journal of Colloid and Interface Science, vol. 355, no. 1, pp. 96-105, 2011.

[32] M. A. A. Razali and A. Ariffin, "Polymeric flocculant based on cassava starch grafted polydiallyldimethylammonium chloride: flocculation behavior and mechanism," Applied Surface Science, vol. 351, pp. 89-94, 2015.

[33] Y. Xiong, X. Huang, B. Lu et al., "Acceleration of floc-water separation and floc reduction with magnetic nanoparticles during demulsification of complex waste cutting emulsions," Journal of Environmental Sciences (China), vol. 89, pp. 80-89, 2020.

[34] R. Lakshmanan and G. Kuttuva Rajarao, "Effective water content reduction in sewage wastewater sludge using magnetic nanoparticles," Bioresource Technology, vol. 153, pp. 333-399, 2014.

[35] J. Ma, K. Fu, X. Fu et al., "Flocculation properties and kinetic investigation of polyacrylamide with different cationic monomer content for high turbid water purification," Separation and Purification Technology, vol. 182, pp. 134-143, 2017.

[36] Y. Chen, S. Liu, and G. Wang, "A kinetic investigation of cationic starch adsorption and flocculation in kaolin suspension," Chemical Engineering Journal, vol. 133, no. 1-3, pp. 325-333, 2007. 